\title{
Prosthodontic stents - A bulwark against radiation
}

\author{
Farhat Jabeen $^{1, *}$, Rajeev Gupta ${ }^{2}$, Aman K Angra ${ }^{3}$, Nitin Sharma ${ }^{4}$, Mrinal Jindal ${ }^{5}$ \\ ${ }^{1,3,4}$ Post Graduate Student, ${ }^{2}$ Professor, ${ }^{5}$ Under Graduate Student, Dept. of Prosthodontics, Himachal Dental College, Sundar \\ Nagar, Himachal Pradesh, India
}

*Corresponding Author:

Email: farhatraza27@gmail.com

\begin{abstract}
As oral malignancies are getting common day by day. Radiotherapy is the prime treatment in most of the case. Unfortunately, these therapies affect the healthy tissue mostly due to lack of any shield. It cannot focus on the tumor or defect most of the time. And also for dentist's it creates hurdle while performing other dental treatment. This article explains few methods and techniques which includes splints and shield to cover, displace and position tissues in an effort to minimize postradiation morbidity. These stents and shiels are usually made of acrylic resins and used prior to radiotherapy. It also awares us about the collaboration of a head and neck specialist and a prosthodontist to save the healthy tissue without any hurdle.
\end{abstract}

Keywords: Prosthodontic stents, Radiotherapy, Positioning stents, Radiation protection stents, Acrylic stents.

\section{Introduction}

In India, one of the most common malignancies are oral malignancies. What's more, there are patients with disease of the nose, nasopharynx, paranasal sinuses and the oropharynx, where treatment includes the oral cavity and in addition the head and neck territory. Radiotherapy is progressively being utilized as an adjunctive type of treatment in the administration of head and neck tumor. Radiation treatment is characterized as "the helpful utilization of ionizing radiation in the administration of neoplasms of the body without medical procedure, or as an adjunctive palliative treatment after medical procedure, either in blend with or without chemotherapy." ${ }^{1}$

The two major orders of ionizing radiations which are used are electromagnetic radiations (photons, $\mathrm{x}$ pillars, gamma shafts) and particulate radiations (electrons, neutrons and protons). The technique for radiotherapy which is used is outside radiation treatment which is in like manner called as teletherapy which is used to pass on high estimations of radiation to tumors that are arranged inside $6 \mathrm{cms}$ of the skin surface. The estimations are 6500 rads to 7500 rads for 6-7 weeks. Interstitial Radiotherapy, also called as Brachytherapy isused to pass on high measurements (upto 20000 rads) of radiation over a short partition for a concise time span period (10-15 hrs). Radiation treatment is given in a movement of meds or divisions called fractionation. Unfortunately, this treatment causes complexities by growing the grimness of the enveloping tissues. These fuse erythema, mucositis, ulcers, parasitic ailments, xerostomia, caries from lessened salivary stream and $\mathrm{pH}$ changes, possible results of pollution in the jaws or the potential for osteoradionecrosis from sullying or damage to lit bone. Unnecessary trickiness of the teeth, taste incident, oral bacterial move and periodontal breakdown are distinctive issues of concern while treating the patient with radiotherapy. ${ }^{2,3}$ Damage to the commonplace tissues can be diminished by using natural methods, for instance, a fitting system for radio treatment and by modifying the dose and fractionation organization. Diverse physical systems are furthermore for the most part used to lessen hurt, which fuse securing, fitting arranging and the usage of various As a preventive measure, radiotherapy guarded contraptions/stents can be made and used in the midst of the treatment. These devices are used to unstick the position or to shield tissues or to help the profitable association of radiotherapy to the affected districts, thus compelling the post treatment horridness. ${ }^{4-6}$ The prerequisite for a radiation stent is controlled by the treating radiotherapist. The prosthodontist can viably help in the rebuilding of danger patients by assembling a whole display of possible prostheses that can be worked to meet specific patient needs, along these lines compelling complexities following therapy. ${ }^{7}$ Radiation stents are frequently made from acrylic pitch and may contain an ensuring metal/compound, dependent upon a couple of conditions; sort of the radiation given, condition of the unfortunate hard and fragile tissues, mouth opening limit and the prerequisites of the treating radiotherapist. Customary prosthetic techniques are used to fabricate these stents. ${ }^{8,9}$ Silicone is the other material of choice. With the types of progress in technology, computer tomography is starting late being used for the fabricate of brachytherapy bearers. Diverse sorts of radiation stents that can be produced are depicted here to entirety things up.fields.

Definition: Stents are appliances constructed to cover the tissues and/or the teeth for their protection; to carry medicaments and/or radium material; to control bleeding or to guide the insertion of dental implants. ${ }^{14}$

\section{Uses}

1. As a guide for radiographic assurance of the best forthcoming site for dental inserts. 
2. As a careful format for directing the inclusion of dental inserts.

3. Protect the tissues after skin and mucosal unions.

4. Keep skin and mucosal unites set up until their revascularization.

5. Prevent sullying of the injuries and advance mending.

6. Preserve the profundity of the vestibules after vestibuloplasty and edge growth.

7. Help to control bleeding, particularly in hemophiliacs.

8. Carry and keep medicaments and careful packs to required territories in the oral.

9. Carry radium materials and reduces the chance of exposure to healhy tissue. ${ }^{14}$

\section{Materials Used:}

1. Acrylic resin: Whether heat-curing or self-curing resins, they may belined with soft materials.

2. Soft materials: Soft rubber or soft resins are used for special purposes. It can also be fabricated from silicon vinyl rubber but an additional reinforcement of resin is usually required.

3. Modeling plastic, black gutta percha and silicone: In emergency cases where there is no time to construct a stent, these materials may be used directly in the oral cavity on the bleeding area.

\section{Intra Oral Stents:}

\section{Surgical Stents}

1. Stents for dental implantation.

2. Surgical stents used after removal of exostosis.

3. Surgical stents used in conjunction with Vestibuloplasty.

4. Stent for epulis fissuratum.

5. Stent for resected maxilla.

6. Palatal stent.

7. Antihaemorrhagic stents.

8. Cyst plugs.

9. Drainage stent.

\section{Extraoral Stents:}

1. Oro- Facial Plug

2. Stents for nasal defect

3. Auditory Stents

4. Burn Stent

5. Stent for neck scar

Intra Oral Stents

\section{1) Stents for Dental Implantation}

Radiographic stent: used as a guide during radiographic determination of the best prospective site for dental implants.

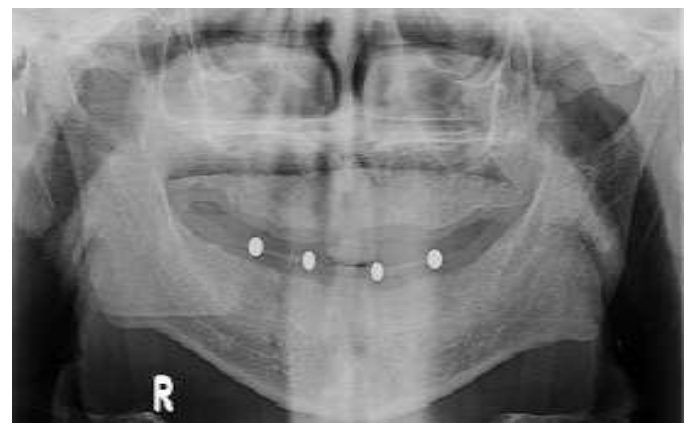

Fig. 1:

Surgical guide stent: used for guiding dental implant during insertion into a predetermined site.

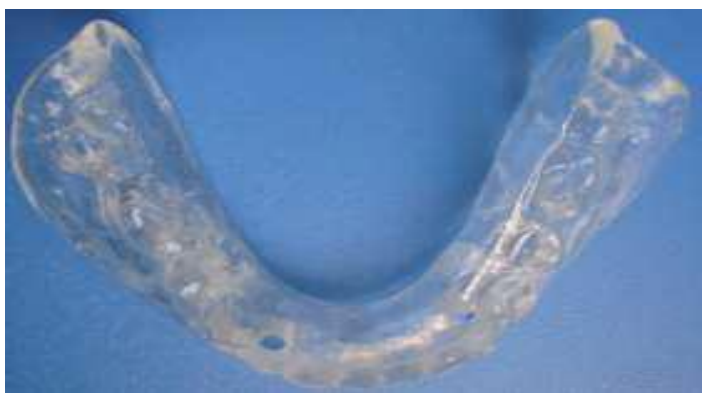

Fig. 2:

Steriolethographic stent for guiding dental implant during insertion into a site predetermined using conebeam computed tomography.

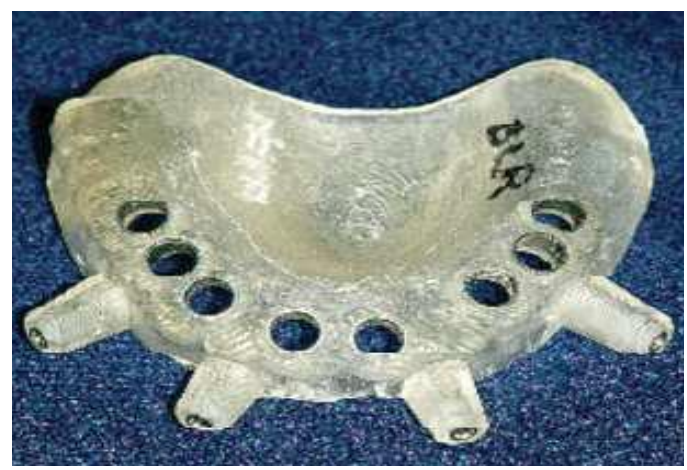

Fig. 3:

Surgical palatal stents used after removal of exostosis: Simple acrylic plate covering the palate after surgery to:

1. Facilitates heamostasis,

2. Protects the healing of raw surface, and

3. Apply pressure to soft tissue to facilitate healing and prevent collapse.

It is constructed before surgery on modified maxillary cast. 


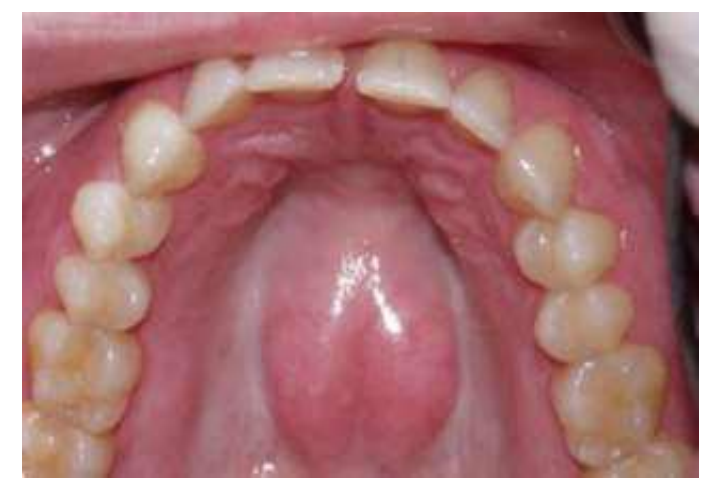

Fig. 4:

Surgical stents used in conjunction with vestibuloplastyand ridge augmentation procedures: ${ }^{14}$ Careful stents utilized as a part of conjunction with Vestibuloplasty (careful extending of the mucobuccal vestibule) and for holding mucous layer and skin joins. This stent is additionally utilized with edge increase strategies to keep up the edge tallness. $^{5}$

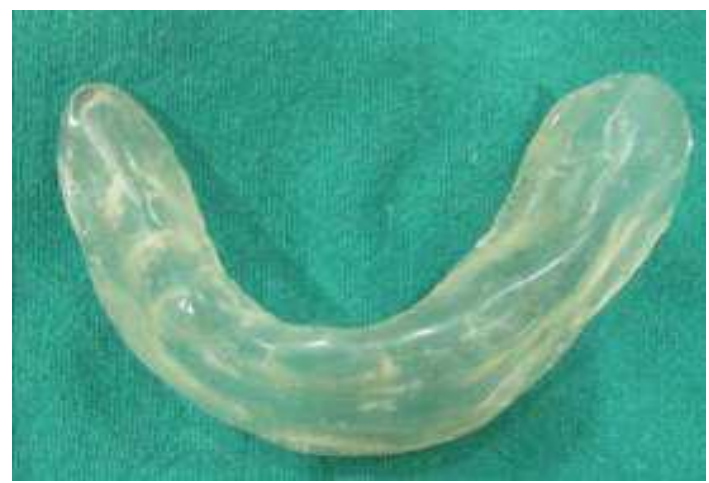

Fig. 5:

\section{Antihaemorrhagic Stents: \\ Definition}

A partial acrylic plate used to control bleeding by applying gentle pressure to the extraction wound.

\section{Intraoral Radiotherapy Stents: \\ Importance of Radiotherapy Stents}

1. It reduces the side effects of the treatment

2. It protects tissues that are not meant to receive radiation.

3. It is safer for the radiotherapist to treat this patient.

4. It increases the accuracy of radiation source and assures that the area of treatment is the same every time the patient is irradiated.

5. It results in a more cooperative patient since discomfort and injuries are reduced.

\section{Types of Radiotherapy Stents}

1. Fluoride carrier (Fluoride stent)

2. Positioning stent or prosthesis to displace the tissues
3. Peroral cone positioning devices or beam locator or beam director (Docking device):

4. Positioning radioactive sources or carrier or applicator:

5. Shielding or tongue protectors

Radiation Source Carriers: This kind of prostheses is required when radiation treatment is to be regulated to limited territories by methods for containers, dabs, tubes or needles of radiation transmitting materials. The principle motivation behind these prostheses is to hold the radiation source safely in a similar place amid the whole time of treatment. It ought to be anything but difficult to stack and empty. The correct area and the quantity of sources are controlled by the radiotherapist and are set apart on the dental model.They are utilized to convey the radiation sources near the site of treatment (intracavitary) or specifically into the tumor (interstitial). ${ }^{10}$ They are of two kinds; preloaded bearers and after stacked transporters.

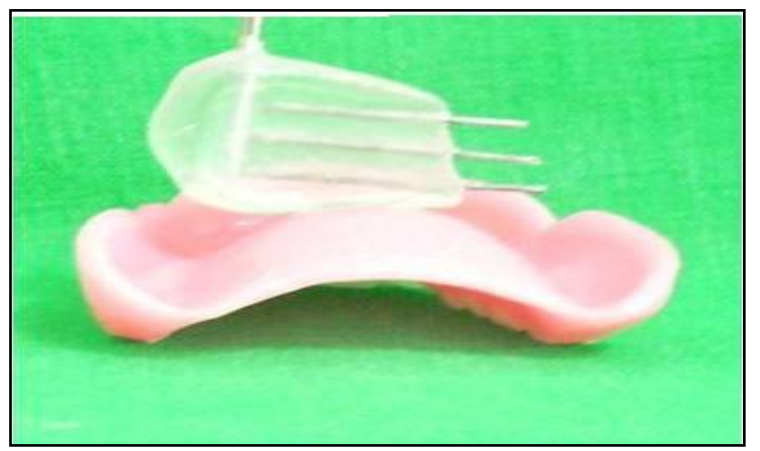

Fig. 6: Preloaded carriers

Perioral Cone Positioning Stents: This kind of stent is usually utilized while boosting the measurement to the injury site. It holds the cone in the repeatable and the correct position as wanted by the radiotherapist, in this manner limiting the odds of the development of the cone amid a specific treatment session. It is utilized as a part of the treatment of shallow sores including the front floor of the mouth and the hard and delicate sense of taste. The real cone or barrel of an indistinguishable distance across from the cones is utilized to frame an acrylic pitch ring of 5 to $6 \mathrm{cms}$ in length. Tin thwart is folded over the cone as a separator from acrylic sap. Within the sight of a radiotherapist, the chamber is appended to the maxillary record base (edentulous patient) or occlusal files (dentulous patients) and the cone is focused over the injury. The treatment cone is embedded into the situating stent for check of the position. 


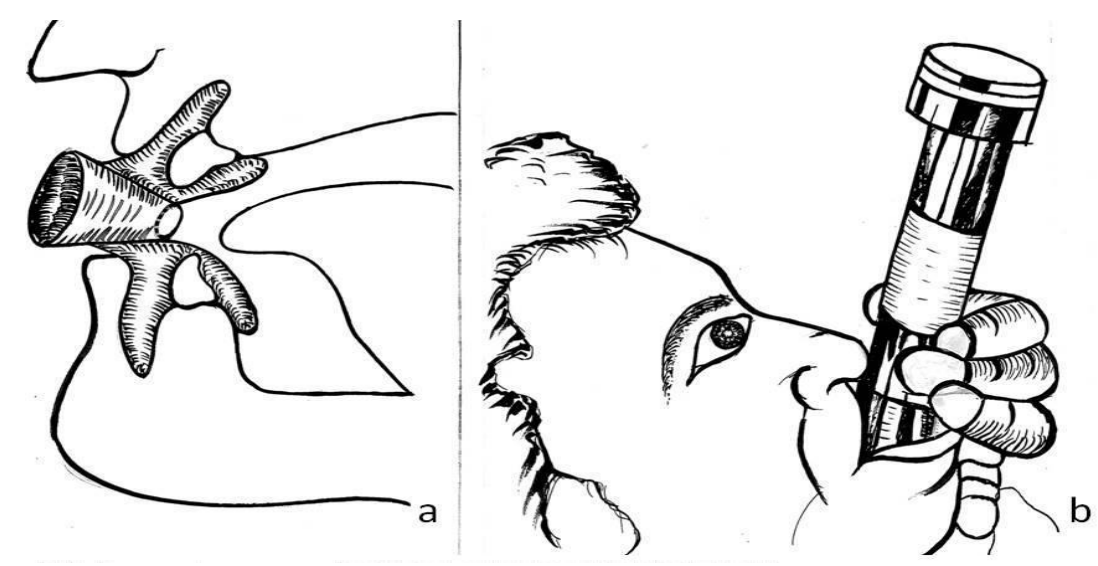

Fig. 7: (a. Perloral cone positioning stent; (b. Radiation cone positioning stent

Radiation Protection/ Shielding Stents : They are utilized to shield the crucial structures which are contiguous radiation treatment locales from overabundance dose of radiation. At the point when electron pillar treatment is utilized to treat injuries of the buccal mucosa, skin or the alveolar edge, mucositis is every now and again saw in regions which are neighboring the metallic dental rebuilding. The treatment shaft dissipates electrons from the high- $Z$ metals utilized as a part of the dental compounds, bringing about a nearby measurements upgrade, which prompts abundance dosage in the encompassing tissues, accordingly causing mucositis. Compelling shields can be manufactured to ensure the tongue, salivary organs and the contrary side of the mandible. ${ }^{11}$ Low dissolving composites like Cerroband, $\mathrm{Pb}-\mathrm{Bi}-\mathrm{Sn}$, and Lipowitz are utilized as protecting materials. Cerrobend amalgam is favored due to its low liquefying temperature and it adequately keeps the transmission of the electron bar. Maxillary and mandibular impressions are made, taking consideration to dislodge the tongue away as an afterthought for which the stent is to be fitted, to make space between the tongue and the alveolar edge. Throws are mounted in driven connection. A lingual augmentation of wax is made in the space made, which is burrowed out to make a cavity which is $1 \mathrm{~cm}$ thick. The wax design is acrylized, completed and cleaned. Cerrobend amalgam is warmed and filled the empty depression and it is fixed with auto polymerizing gum to counteract back disseminate measurement upgrade.

Position Maintaining Stents: It is utilized to unequivocally position structures which are to be dealt with in settled and repeatable positions for different treatment sessions. They are utilized to position versatile structures like tongue, delicate sense of taste and so forth.

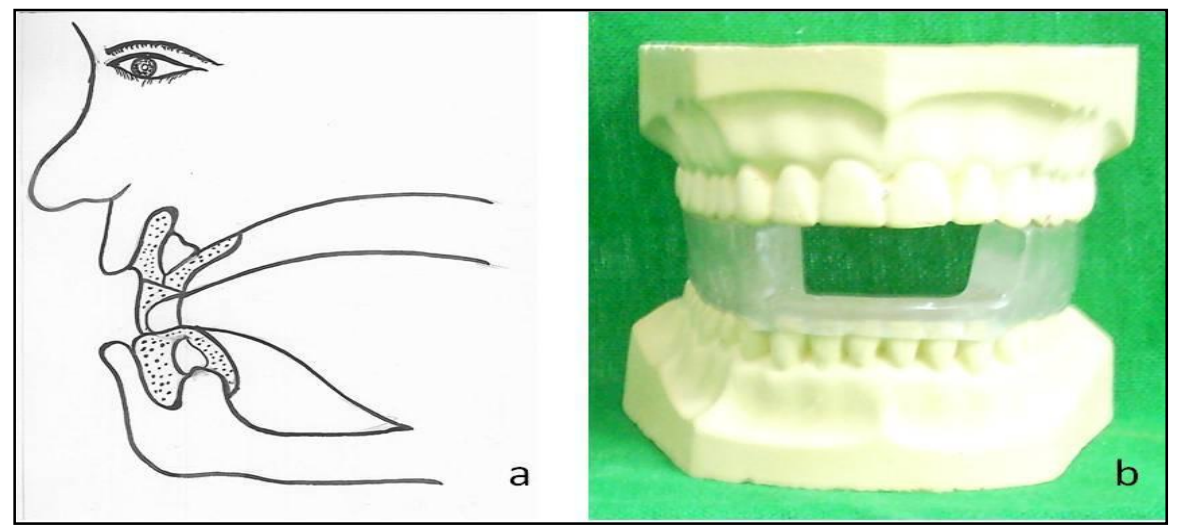

Fig. 8: Posltion maintaining stent

Tongue Depressing Stents: It is a specially crafted gadget which positions the mandible, discourages the tongue and extras the parotid organ amid radiotherapy of head and neck tumors. These stents are more precise and give more noteworthy patient solace than the ordinarily utilized "Plug and tongue cutting edges". An interocclusal stent is set up for the dentate patient, that expands lingually from both the alveolar edges, with a level plate of acrylic sap which serves to discourage the tongue. A gap is made in the front portion in which the tip of the tongue is submitted in request to build up a reproducible position. 


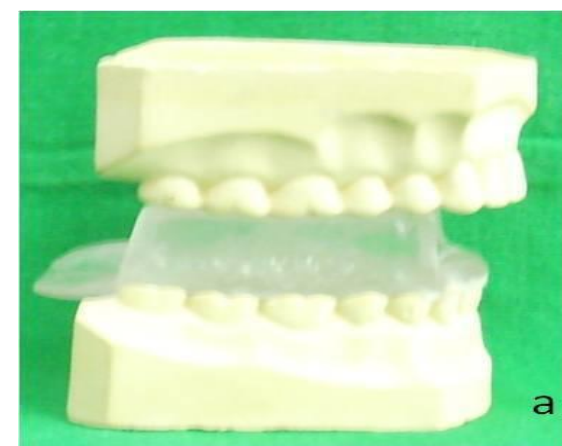

Fig: 9: Tongue depressing stent

Dosimeter Positioning Stents: Dosimeter is a device which is utilized to compute the measure of dosages required for a sore. These stents are composed with a progression of openings for holding lithium fluoride containers. Lithium fluoride containers utilized as a dosimeter are a precise and effective methods for

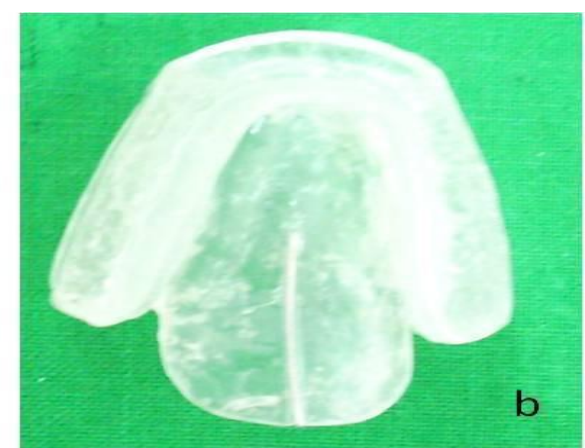

deciding measurements locally. The lithium fluoride case is enveloped by a 0.1 inch tinfoil which is wrapped with acrylic gum packaging and is permitted to cure. A gap is set toward one side of the stent and an orthodontic wire is utilized to drive the case out of the acrylic sap packaging. The pitch case is joined to the stent in position as coordinated by the radiotherapist.

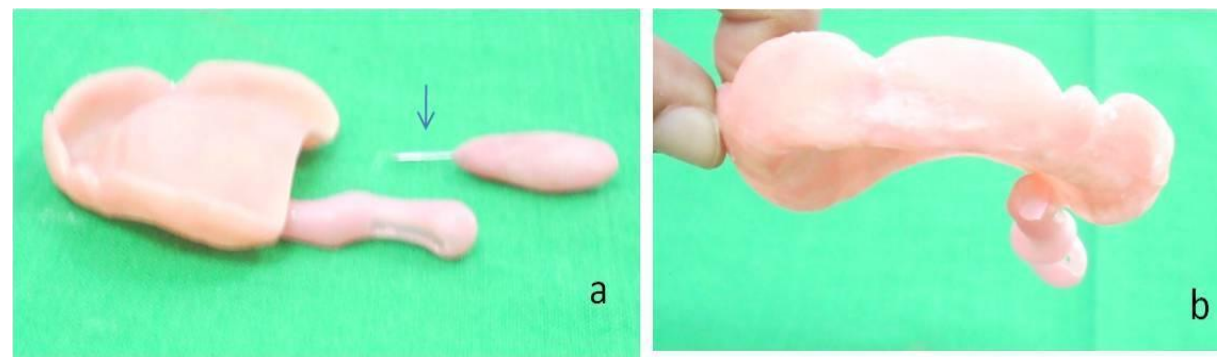

Fig. 10: Dosimeter positioning stent; (a. Lithium fluoride carrier attached to maxillary base; (b. Lithium flouride carrier

Tissue Recontouring Stents: These stents are successful while treating skin injuries which are related with lips when the bar is balanced for midlines. Low measurements are conveyed at the edges of the mouth on account of the ebb and flow of the lips. A stent can be made to smooth the lip and the edge of the mouth, in this manner putting the whole lip in a similar plane. These stents are created by displaying plastic and are handled in acrylic rest.

Tissue Bolus Compensators/ Balloon Bolus Supporting Stents: These prostheses help in the treatment of shallow sores of the face with unpredictable forms. Because of anomalies in the injuries, a few territories inside the field might be untreated, while others may create confined hotspots. BOLUS is a tissue proportional material which is set straightforwardly onto or into the abnormalities, that aides in changing over unpredictable tissue forms into level surfaces which are opposite to the focal pivot of the ionizing bar, to along these lines all the more precisely help in the homogenous appropriation of the radiation. ${ }^{12,13}$ The most normally utilized materials for bolus are tissue conditioners, water, saline, waxes and acrylic tar.

\section{Conclusion}

Apart from prosthodontic uses, numerous oral complication related with radiotherapy can also be controlled with the treatment prosthesis given by prosthodontist. On occasion, the head and neck specialist, radiotherapist are not aware of numerous essential and strong administration that the maxillofacial prosthodontist can perform using these types of prosthesis. It is recommended that such a specialist be on the team for consultation before planning any head and neck cancer surgery or before starting radiotherapy. These measures make the patient's treatment course smoother and simplify the surgeon's treatment plan.

\section{References}

1. Beumer J, Curtis TA. Firtell D N Radiation therapy of head and neck tumors: oral effects and dental manifestation. In maxillofacial Rehabilitation (Prosthodontic \&amp; Surgical Consideration). The CV Mosby Company, 1979. Chp.3, pg. 23.

2. Chalian VA, Drane J B Standish SM. Splints and stents. In maxillofacial Prosthetics Multidisciplinary practice. The Williams \& amp; Williams Co. Baltimore 1971. Chap. 15, pg. 234,

3. Kramer DC. The Radiation Therapy patient treatment planning and post treatment care. In Taylor TD, editors Clinical Maxillofacial Prosthetics. Quintessence Publishing Co. Inc. 2000. Chapter 3, pg 37. 
4. Kaanders JH, Fleming TJ, Ang KK, Maor MH, Peters LJ. Devices valuable in head and neck radiotherapy. Into $J$ Riadial Once Boil Phys 1992;23(3):639-45.

5. Chambers MS, Tooth BB, Fleming TJ, Lemon JC. Oral and Dental Management of the cancer patient: prevention and treatment of complications. Support Care Cancer 1995;3:168-75.

6. Sheaf NG. Maxillofacial prosthetics and head and neck cancer patients. Cancer 1984;54:2682-90.

7. Palates J, Gilliam KK. Oral care protocols for patients undergoing cancer therapy. Gen Dent July-Aug 2008; 6478.

8. McCarthy D, Omer O, Nunn J and Cotter F. Oral health needs of the Head and Neck Radiotherapy Patients: Epidemiology, effects of Radiotherapy and role of the GDP in Diagnosis. Dent update 2005;32:512-22.
9. Prostheses, stents and splints for the oral cancer patient. Jeri FC, Ramsey WO, Drone JB, et al. In oral care for the oral cancer patient, public health service. Pub No. 1968;341-52.

10. Kabcenell JL. A two piece radiation therapy surface mould. J. Prosthet Dent 1980;43:86-88.

11. Wang RR, Olmsted LW. A direct methods for fabricating tongue shielding stent. J Prosthet Dent 1995;74:171-73.

12. Miyamoto RH, Fleming TJ, Davis MG. Radio therapeutic management of an Orocutaneous defect with a balloon retaining stent. J. Prosthet Dent 1992;68:115-17.

13. Brosky M, Lee C, Barlett T, Lo S. Fabrication of radiation bolus prosthesis for the maxillectomy patient. $J$ Prosthet Dent 2000;83:119-20.

14. Mohammed M Fouad, Guidelines For Maxillofacial Prosthesis, Stents book. 